\title{
Molecular Dynamics Analysis of Grain-Boundary Grooving in Thin-Film Interconnects for ULSIs
}

\author{
Tomio Iwasaki and Hideo Miura \\ Mechanical Engineering Research Laboratory, Hitachi, Ltd. \\ 502 Kandatsu, Tsuchiura, Ibaraki 300-0013, Japan
}

\begin{abstract}
Grain-boundary grooving in an Al thin film was analyzed by molecular-dynamics simulation. The simulation result showed that the groove was formed by atom transport due to diffusion at the intersection of the grain-boundary and free surface. The effect of impurity on groove formation was analyzed in terms of atomic radius and bond energy. It was found that when the atomic radius of an impurity is smaller than that of $\mathrm{Al}$ and when $\mathrm{Al} /$ impurity bond energy is close to $\mathrm{Al} / \mathrm{Al}$ bond encrgy, groove formation is prevented due to the suppression of the diffusion. This diffusion suppression is caused by large cohesive energy and small interatomic distance.
\end{abstract}

\section{Introduction}

The problem of migration-induced open failures in thin-film interconnects is one of the major problems to be solved in order to improve product reliability of ULSIs. The migration is caused not only by an electrical field but also by the gradient of a mechanical-stress field. Grain-boundary grooving due to diffusion is a dominant factor in the migration [1], [2]. Conventionally, impurity doping, such as $\mathrm{Cu}$ doping into Al films [3], [4], is known to be an effective method for preventing the diffusion of the principal-element atoms of thin films ( $\mathrm{Al}$ atoms, for example). However, such an effective impurity element has been found only by experimental trial and error. There has been no theoretical explanation of how the impurity reduces the diffusion that causes the transport of the principal-element atoms of thin films. The authors have therefore developed a molecular-dynamics simulation technique that analyzes grain-boundary grooving and diffusion in thin films. The effect of impurity on groove formation and diffusion was investigated using this simulation technique.

\section{Analysis Method}

'The basic equations used in our simulation technique are Newton's well known equation of motion, given as

$$
\mathrm{m}_{1} \mathrm{~d}^{2} r_{\mathrm{i}} / \mathrm{d} t^{2}=-\partial \sum_{i<j} u\left(\left|r_{i}-r_{j}\right|\right) / \partial r_{1},
$$

where $m_{1}$ is the atomic mass of the $\mathrm{i}$-th atom and $u\left(\left|r_{1}-r_{\mathrm{j}}\right|\right)$ is interatomic potential 
between the $\mathrm{i}$-th and $\mathrm{j}$-th atoms located at $\boldsymbol{r}_{1}$ and $\boldsymbol{r}_{\mathrm{J}}$, respectively. In this paper, this potential is written as the Morse type potential:

$$
u\left(r_{\mathrm{ij}}\right)=-u_{\min }\left\{\exp \left[-2 b\left(r_{\mathrm{ij}}-2 r_{\mathrm{m}}\right)\right]-2 \exp \left[-b\left(r_{\mathrm{y}}-2 r_{\mathrm{m}}\right)\right]\right\} . \cdots \cdots
$$

Here, $u_{\min }, a$, and $b$ are listed in reference [5]. (e.g., for Al, $u_{\min }=-19.1149 \times 10^{21} \mathrm{~J}$, $r_{\mathrm{m}}=0.143247 \mathrm{~nm}$, and $b=23.53643 \mathrm{~nm} \mathrm{'}$; for $\mathrm{Cu}, u_{\mathrm{m} \text {. }}=-28.8382 \times 10^{21} \mathrm{~J}, r_{\mathrm{m}}$ $=0.128532 \mathrm{~nm}$, and $b=23.27429 \mathrm{~nm}{ }^{1}$ ). The equations were numerically solved by using the Verlet algorithm [6] with the time step increment $h=3.0 \times 10^{15} \mathrm{~s}$.

An Al thin film with a grain boundary was used as a computation model (Fig. 1). In the simulation, atoms at the top surface were free to move while those in the bottom layer were fixed. By heating the Al thin film to $600 \mathrm{~K}$, we simulated grain-boundary grooving.

To investigate the relationship between diffusion and grain-boundary grooving, we divided the computation model into Regions $B, G, S$, and $C$ (Fig. 2), and calculated the corresponding diffusion coefficients $D_{B}, D_{G}, D_{s}$, and Dc. Regions $B, G$, and $S$ represent bulk, the grain boundary, and free surface, respectively. Region $C$ represents the intersection of the grain boundary and surface. When there are no impurity atoms, we obtained the following diffusion coefficients: $D_{B}=0.0028 \times 10^{-10}$ $\mathrm{m}^{2} / \mathrm{s}, D_{\mathrm{c}}=0.024 \times 10^{-10} \mathrm{~m}^{2} / \mathrm{s}, D_{\mathrm{s}}=0.32 \times 10^{-10} \mathrm{~m}^{2} / \mathrm{s}$, and $D_{c}=4.6 \times 10^{-10} \mathrm{~m}^{2} / \mathrm{s}$. Because diffusion in Region $\mathrm{C}$ was dominant, we focused on $\mathrm{Dc}_{\mathrm{C}}$ and positioned an impurity atom at the center of Region $\mathrm{C}$.

\section{Analysis Results}

Typical simulation results are shown for three conditions: with no impurity, with a $\mathrm{Cu}$ atom, and with a $\mathrm{Cr}$ atom. For each condition the system reached equilibrium at about Step 200,000. We show the atomic configurations at Step 210,000 in Figs. 3 (a), (b), and (c). When there were no impurity atoms, atom transport occurred at the intersection of the grain boundary and the free surface, and a groove about $1 \mathrm{~nm}$ deep was formed as shown in Fig. 3 (a). When a $\mathrm{Cu}$ atom was present, the groove formation was prevented (Fig. 3 (b)). On the other hand, when a $\mathrm{Cr}$ atom was present, groove formation was activated (Fig. 3 (c)).

To clarify what kind of impurity is useful for preventing the grain-boundary groove formation, we used another four kinds of impurities ( $\mathrm{Mg}, \mathrm{Au}, \mathrm{Zn}$, and $\mathrm{Zr}$ ) as well as virtual impurities whose atomic radius and bond energy can be varied. The interatomic potential between a virtual impurity atom and an $\mathrm{Al}$ atom was assumed to be represented by the Morse-type potential (i.e., eqn. (1)). By varying atomic radius $\left(r_{\mathrm{m}} / 2\right)$ and bond energy $\left(\left|u_{\mathrm{min}}\right|\right)$, we investigated how the groove depth depends on the atomic radius and bond energy. The result is shown in Fig. 4. This figure shows that when the atomic radius of an impurity is smaller than that of $\mathrm{Al}$ and when the $\mathrm{Al} / \mathrm{impurity}$ bond energy is close to the $\mathrm{Al} / \mathrm{Al}$ bond energy, the groove depth is reduced. The dark area shows the region of impurity that reduces the groove depth by $50 \%$. Copper is the most useful impurity in preventing groove formation in Al films, and this result is consistent with the published experimental results [3], [4]. 


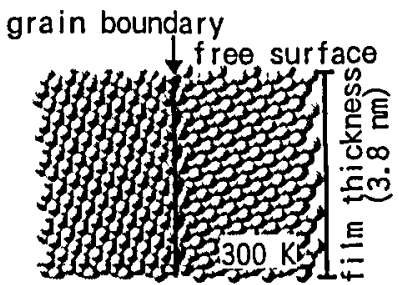

Figure 1: Computation model.

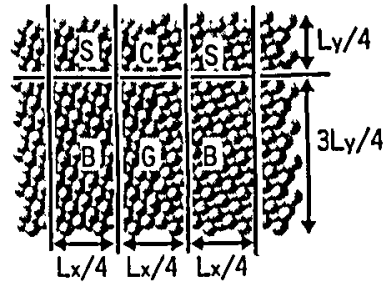

Figure 2: Regions C, S, G and B.

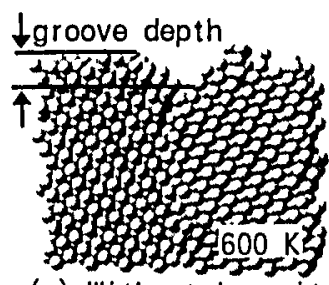

(a) Wi thout impurity

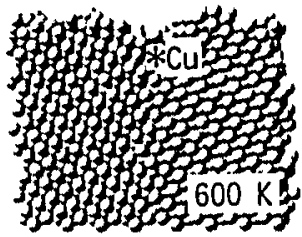

(b) With a $\mathrm{Cu}$ atom

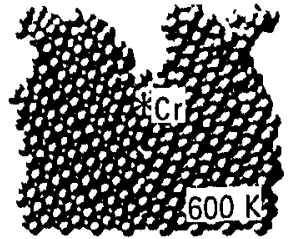

(c) Wi th a $\mathrm{Cr}$ atom

Figure 3: Grain-boundary groove in an $\mathrm{Al}$ film.

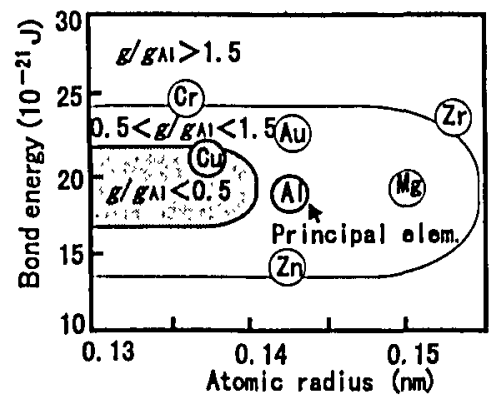

Figure 4: Impurity effect on the groove depth $g$ (gAl: groove depth in the absence of impurity).

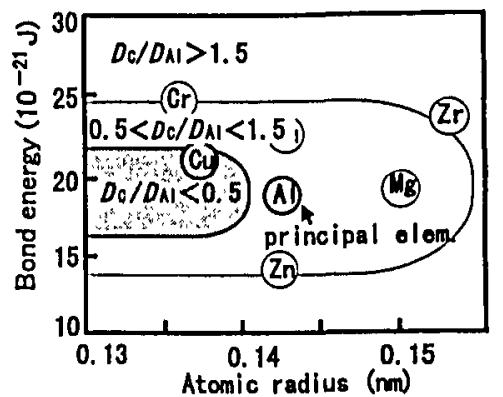

Figure 5: Impurity effect on the diffusion coefficient $D \mathrm{c}$ in Region $\mathrm{C}\left(D_{\mathrm{A}}\right.$ : diffusion coefficient in the absence of impurity).

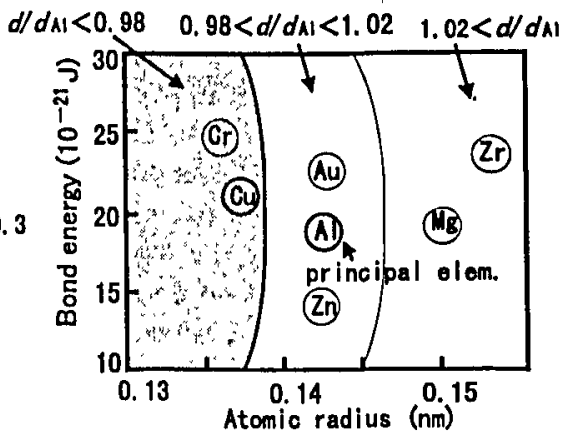

Figure 6: Impurity effect on the averaged cohesive energy $U$ in Region $\mathrm{C}\left(U_{N}\right.$ : averaged cohesive energy in the absence of impurity)

Figure 7: Impurity effect on the averaged interatomic distance $d$ in Region C (dN: averaged interatomic distance in the absence of impurity) 
Thus our simulation technique is useful for finding effective impurities which prevent the grain-boundary groove formation, i.e., migration-induced failure.

To investigate the relationship between groove formation and diffusion, we calculated the dominant diffusion coefficient, $D$ c. The result for the impurity effect on $D_{\mathrm{c}}$ is shown in Fig. 5 . The pattern of contour lines for $D_{\mathrm{c}}$, shown in Fig. 5, fits that for the groove depth (Fig. 4). So it was found that the suppression of diffusion in Region $\mathrm{C}$ is the key to preventing groove formation. The dark areas in Figs. 4 and 5 show that to reduce the groove depth by $50 \%$, we must also reduce $D$ c by $50 \%$. The dominant factors of diffusion in Region $\mathrm{C}$ are considered to be the averaged cohesive energy ( $U$ ), which describes the energy needed to break a bond between $\mathrm{Al}$ atoms in Region $\mathrm{C}$, and the averaged interatomic distance $(d)$, which shows how easily $\mathrm{Al}$ atoms move. So we investigated the impurity effect on these factors. The effect of impurity on $U$ is shown in Fig. 6. When $\mathrm{Al} /$ impurity bond energy is close to $\mathrm{AV} / \mathrm{Al}$ bond energy, $U$ is large, which means that bonds between $\mathrm{Al}$ atoms are not easily broken. The effect of impurity on $d$ is shown in Fig. 7 . When the atomic radius of an impurity is smaller than that of $\mathrm{Al}, d$ is small, which means that it is difficult for $\mathrm{Al}$ atoms to move. The dark areas in Figs. 5, 6, and 7 show that to reduce $D$ c by $50 \%$, the cohesive energy and the interatomic distance must satisfy $U / U_{A l}>0.6$ and $\mathrm{d} / \mathrm{d}_{\mathrm{Nl}}<0.98$, where $U_{\mathrm{N}}$ and $d_{\mathrm{Al}}$ are values in the absence of impurity.

\section{Summary}

We developed a molecular-dynamics simulation technique that analyzes grain boundary grooving and diffusion in a thin film, and we applied this technique to an Al thin film. The simulation results showed that a groove was formed due to diffusion at the intersection of the grain boundary and the free surface. The effect of impurity on groove formation and diffusion was analyzed in terms of atomic radius and bond energy. It was found that when the atomic radius of an impurity is smaller than that of $\mathrm{Al}$ and $\mathrm{Al} / \mathrm{impurity}$ bond energy is close to $\mathrm{Al} / \mathrm{Al}$ bond energy, groove formation is prevented due to the suppression of the diffusion. This diffusion suppression is caused by large cohesive energy and small interatomic distance.

\section{References}

[1] 'T. Kitamura et al., Trans. Jpn. Soc. Mech. Eng., Vol. 59, No. 563, A(1993), 1625.

[2] N. Owada, et al., Proc. IEEE 2nd Int. VLSI Multilevel Interconnection Conference, (1985), 173.

[3] T. Turner, et al., Proc. Int. Reliability Physics Sym., Orland, (1985), 142.

[4] S. Mayumi, et al., Proc. Int. Reliability Physics Sym., San Diego, (1987), 15.

[5] A. M. Stohnham et al., Handbook of Interatomic Potentials, Vol. 2, (1981).

[6] L. Verlet, Phys. Rev., Vol. 159, (1967), 98. 